\title{
HOW TO RECOGNIZE A LOCALIZED SPHERE
}

\author{
J. AGUADÉ
}

\begin{abstract}
Two cohomological characterizations of the sphere localized at a prime are given.
\end{abstract}

The purpose of this note is to obtain necessary and sufficient conditions on the cohomology of a nilpotent space in order to insure that it has the homotopy type of $S_{p}^{m}$, the sphere localized at the prime $p$ (cf. [2]). $p$ will denote a fixed prime integer. $\hat{\mathbf{Z}}_{p}, \mathbf{Z}_{(p)}, \mathbf{Z}_{n}, \mathbf{Z}_{p^{\infty}}$ denote the $p$-adic integers, the integers localized at $p$, the integers modulo $n$ and the $p^{m}$ th roots of unity with $m$ running over all integers $>0$, respectively. Group will always mean abelian group. A group is called $p$-local if it is $q$-divisible for every $q \neq p$ and has no $q$-torsion for every $q \neq p$. A nilpotent space is called $p$-local if its homology groups with integral coefficients are $p$-local $[2, \mathrm{p}$. 53].

Recall that a subgroup $B$ of a group $A$ is called $p$-basic if the following holds:

(i) $B$ is a direct sum of cyclic $p$-groups and infinite cyclic groups,

(ii) $A / B$ is $p$-divisible,

(iii) $B / p^{k} B$ is a direct summand of $A / p^{k} B$ for all $k$.

Every group contains $p$-basic subgroups [1, I, p. 137].

We use freely the most elementary properties of the functors Hom and Ext. A standard reference is [1, Chapters VIII and IX]. In particular we shall use the following:

$\operatorname{Ext}\left(\mathbf{Z}_{n}, A\right)=A / n A, \operatorname{Ext}\left(\mathbf{Z}_{p^{\infty}}, \mathbf{Z}_{p}\right)=\mathbf{Z}_{p}, \operatorname{Ext}\left(\mathbf{Q}, \mathbf{Z}_{(p)}\right)=\mathbf{Q}^{\aleph_{0}}, \operatorname{Ext}\left(\mathbf{Z}_{p^{\infty}}, \mathbf{Z}_{(p)}\right)=\hat{\mathbf{Z}}_{p}$.

All spaces are assumed to be of the homotopy type of $\mathrm{CW}$-complexes.

Lemma 1. Let $B$ be a p-basic subgroup of $A$. Then $\operatorname{Hom}\left(A, \mathbf{Z}_{p}\right)=\operatorname{Hom}\left(B, \mathbf{Z}_{p}\right)$.

Proof. Since $A / B$ is $p$-divisible and $\mathbf{Z}_{p}$ contains no $p$-divisible subgroups other than 0, the Hom-Ext exact sequence associated to $B \mapsto A \rightarrow A / B$ yields that $\operatorname{Hom}\left(A, \mathbf{Z}_{p}\right)$ is a subgroup of $\operatorname{Hom}\left(B, \mathbf{Z}_{p}\right)$. Let $f: B \rightarrow \mathbf{Z}_{p}$. Clearly, $f$ factorizes through $f^{\prime}: B / p B \rightarrow Z_{p}$. Since $B$ is a $p$-basic subgroup of $A, B / p B$ is a direct summand of $A / p B$. Hence $f^{\prime}$ extends to $A / p B$ and yields a homomorphism $g \in \operatorname{Hom}\left(A, \mathbf{Z}_{p}\right)$.

LemMA 2. If $A$ is a p-local group such that $\operatorname{Hom}(A, \mathbf{Q})=\operatorname{Hom}\left(A, \mathbf{Z}_{p}\right)=0$, then $A$ is a divisible p-group.

Received by the editors January 18, 1979.

AMS (MOS) subject classifications (1970). Primary 55J20, 20K30, $20 \mathrm{~K} 35$.

Key words and phrases. Localization, nilpotent spaces, universal coefficient theorem.

() 1980 American Mathematical Society 0002-9939/80/0000-0179/\$02.00 
Proof. Since $\operatorname{Hom}(A, \mathbf{Q})=0, A$ has no elements of infinite order. Since $A$ is $p$-local, $A$ is a $p$-group. Let $B$ be a $p$-basic subgroup. By $\operatorname{Lemma} 1, \operatorname{Hom}\left(B, \mathbf{Z}_{p}\right)=$ $\operatorname{Hom}\left(A, \mathbf{Z}_{p}\right)=0$ and since $B$ is a direct sum of cyclic $p$-groups then $B=0$. That is $A$ is $p$-divisible and so, since $A$ is $p$-local, $A$ is divisible.

Lemma 3. Let $E$ be a nilpotent space such that $H_{*}(E ; \mathbf{Z})=H_{*}\left(S_{p}^{m} ; \mathbf{Z}\right)$. Then $E$ has the homotopy type of $S_{p}^{m}$.

Proof. If $m>1$ then $H_{1} E=0$. In other words, the abelianization of $\pi_{1} E$ is trivial. Since $\pi_{1} E$ is a nilpotent group we conclude that $E$ is simply connected and so $E$ is a Moore space $M\left(\mathbf{Z}_{(p)}, m\right)$, that is a localized sphere $S_{p}^{m}$.

If $m=1$ then we have

$$
\left[E, S_{p}^{1}\right]=\left[E, K\left(\mathbf{Z}_{(p)}, 1\right)\right] \cong H^{1}\left(E ; \mathbf{Z}_{(p)}\right)=\mathbf{Z}_{(p)}
$$

where $K\left(\mathbf{Z}_{(p)}, 1\right)$ denotes an Eilenberg-Mac Lane space of type $\left(\mathbf{Z}_{(p)}, 1\right)$. Then there exists a map $f: E \rightarrow S_{p}^{1}$ which induces isomorphisms in homology. Because $E$ and $S_{p}{ }^{1}$ are nilpotent spaces, $f$ must be a homotopy equivalence. The result follows.

THEOREM 4. Let $E$ be a nilpotent space such that

(1) $E$ is p-local,

(2) $H^{*}(E ; R)=H^{*}\left(S^{m} ; R\right)$ where $R=\mathbf{Z}_{p}$ or $R=\hat{\mathbf{Z}}_{p}$,

(3) $H^{*}(E ; \mathbf{Q})=H^{*}\left(S^{m} ; \mathbf{Q}\right)$,

(4) $H^{m+1}\left(E ; \mathbf{Z}_{(p)}\right)=0$.

Then $E=S_{p}^{m}$. If any of the above conditions is omitted then the conclusion is false.

Proof. It suffices to prove that $H_{*}(E ; \mathbf{Z})=H_{*}\left(S_{p}^{m} ; \mathbf{Z}\right)$. Let us consider the exact sequence $\hat{\mathbf{Z}}_{p} \stackrel{\cdot p}{\rightarrow} \hat{\mathbf{Z}}_{p} \rightarrow \mathbf{Z}_{p}$. It induces a long exact sequence in cohomology:

$$
\ldots \rightarrow H^{r}\left(E ; \hat{\mathbf{Z}}_{p}\right) \stackrel{\cdot p}{\rightarrow} H^{r}\left(E ; \hat{\mathbf{Z}}_{p}\right) \rightarrow H^{r}\left(E ; \mathbf{Z}_{p}\right) \rightarrow H^{r+1}\left(E ; \hat{\mathbf{Z}}_{p}\right) \rightarrow \ldots
$$

From this it follows easily that we may assume, without loss of generality that $R=\mathbf{Z}_{p}$ in condition (2). On the other hand, condition (3) implies that $H_{r} E$ has torsion-free rank zero if $r \neq m$ and has torsion-free rank one if $r=m$.

The universal coefficient theorem:

$$
\operatorname{Ext}\left(H_{r-1} E, \mathbf{Z}_{p}\right) \mapsto H^{r}\left(E ; \mathbf{Z}_{p}\right) \rightarrow \operatorname{Hom}\left(H_{r} E, \mathbf{Z}_{p}\right)
$$

yields: $\operatorname{Hom}\left(H_{r} E, \mathbf{Z}_{p}\right)=0$ if $r \neq m$ and $\operatorname{Ext}\left(H_{r} E, \mathbf{Z}_{p}\right)=0$ if $r \neq m-1$. It follows from Lemma 2 that $H_{r} E$ is a divisible $p$-group if $r \neq m$. The structure theorem for divisible groups [1, I, p. 104] shows that $H_{r} E=\bigoplus \mathbf{Z}_{p^{\infty}} . \operatorname{But} \operatorname{Ext}\left(H_{r} E, \mathbf{Z}_{p}\right)=0$ if $r \neq m-1$, hence $H_{r} E=0$ if $r \neq m, m-1$ because $\operatorname{Ext}\left(\mathbf{Z}_{p^{\infty}}, \mathbf{Z}_{p}\right)=\mathbf{Z}_{p}$.

Since $H^{m}\left(E ; \mathbf{Z}_{p}\right)=\mathbf{Z}_{p}$ the universal coefficient theorem shows that there are two cases:

(A) $\operatorname{Ext}\left(H_{m-1} E, \mathbf{Z}_{p}\right)=\mathbf{Z}_{p}, \operatorname{Hom}\left(H_{m} E, \mathbf{Z}_{p}\right)=0$.

$H_{m-1} E$ is a divisible $p$-group and so $H_{m-1} E=\bigoplus \mathbf{Z}_{p^{\infty}}$. Since $\operatorname{Ext}\left(H_{m-1} E, \mathbf{Z}_{p}\right)=$ $\mathbf{Z}_{p}$ it follows that $H_{m-1} E=\mathbf{Z}_{p^{\infty}}$. On the other hand, let $B$ be a $p$-basic subgroup of $H_{m} E$. By Lemma $1, \operatorname{Hom}\left(B, \mathbf{Z}_{p}\right)=\operatorname{Hom}\left(H_{m} E, \mathbf{Z}_{p}\right)=0$ and so $B=0$ and $H_{m} E$ is divisible because it is $p$-local and $p$-divisible. Since $H_{m} E$ has torsion-free rank one and $\operatorname{Ext}\left(H_{m} E, \mathbf{Z}_{p}\right)=0$ we conclude that $H_{m} E=\mathbf{Q}$. Hence, we see that in this case 
the space $E$ has the homology of $M(\mathbf{Q}, m) \vee M\left(\mathbf{Z}_{p^{\infty}}, m-1\right)$, the wedge of the Moore spaces $M(\mathbf{Q}, m)$ and $M\left(\mathbf{Z}_{p^{\infty}}, m-1\right)$.

(B) $\operatorname{Ext}\left(H_{m-1} E, \mathbf{Z}_{p}\right)=0, \operatorname{Hom}\left(H_{m} E, \mathbf{Z}_{p}\right)=\mathbf{Z}_{p}$.

$H_{m-1} E$ is a divisible $p$-group such that $\operatorname{Ext}\left(H_{m-1} E, \mathbf{Z}_{p}\right)=0$. Since $\operatorname{Ext}\left(\mathbf{Z}_{p^{\infty}}, \mathbf{Z}_{p}\right)$ $=\mathrm{Z}_{p}$ then $H_{m-1} E=0$ and so $E$ has homology $\neq 0$ only in dimension $m$. Let $B$ be a $p$-basic subgroup of $H_{m} E$. We have $\operatorname{Hom}\left(B, \mathbf{Z}_{p}\right)=\operatorname{Hom}\left(H_{m} E, \mathbf{Z}_{p}\right)=\mathbf{Z}_{p}$. Since $B$ is a direct sum of infinite cyclic groups and cyclic $p$-groups, either $B=\mathbf{Z}$ or $B=\mathbf{Z}_{p^{k}}$. If $B=\mathbf{Z}_{p^{k}}$ then condition (iii) in the definition of $p$-basic subgroup yields that $\mathbf{Z}_{p^{k}}$ is a direct summand of $H_{m} E$, but this is impossible because $\operatorname{Ext}\left(H_{m} E, \mathbf{Z}_{p}\right)$ $=0$. Hence $\mathbf{Z}$ is a $p$-basic subgroup of $H_{m} E$. Since $D=H_{m} E / Z$ is divisible and $\operatorname{Ext}\left(H_{m} E, \mathbf{Z}_{p}\right)=0$, the Hom-Ext exact sequence associated to $\mathbf{Z} \mapsto H_{m} E \rightarrow D$ gives $\operatorname{Ext}\left(D, \mathbf{Z}_{p}\right)=0$. If we apply now the structure theorem for divisible groups we obtain that $D$ has no $p$-torsion and so $H_{m} E$ is torsion-free because it is $p$-local and so it can only have $p$-torsion. Then $H_{m} E$ is a $p$-local torsion-free group of rank one. We apply now the classification theorem for these groups [1, II, p. 110]. Since $H_{m} E$ is $p$-local, in order to prove that $H_{m} E=\mathbf{Z}_{(p)}$ it suffices to show that $H_{m} E$ contains elements of $p$-height zero. Let us consider $1 \in \mathbf{Z} \subset H_{m} E$. If $1=p a$, $a \in H_{m} E$ then in $D$ we have $0=p \bar{a}$ and since $D$ has no $p$-torsion we get $a \in \mathbf{Z}$, a contradiction. Hence, the type of $H_{m} E$ is $t_{q}=\infty$ if $q \neq p$ and $t_{p}=0$. Then the space $E$ has the same integral homology as $S_{p}^{m}$ and then, by Lemma 3, $E=S_{p}^{m}$.

We have proved that if a nilpotent space $E$ satisfies conditions (1), (2), (3) of the theorem then either $E=S_{p}^{m}$ or $E$ has the same homology as $M(Q, m) \bigvee$ $M\left(\mathrm{Z}_{p^{\infty}}, m-1\right)$. It suffices to show that if $H_{m} E=\mathbf{Q}$ then $E$ does not satisfy condition (4). This follows easily from the fact $\operatorname{Ext}\left(\mathbf{Q}, \mathbf{Z}_{(p)}\right)=\mathbf{Q}^{\aleph_{0}}$.

Finally, note that none of the conditions (1), (2), (3), (4) can be omitted. Let us consider the spaces $E_{1}=S_{p}^{m} \vee M\left(\mathbf{Z}_{q}, 2\right)(q$ a prime $\neq p) ; E_{2}=S_{p}^{m} \vee M\left(\mathbf{Z}_{p}, 2 m\right)$; $E_{3}=M\left(\mathbf{Z}_{p^{\infty}}, m-1\right) ; E_{4}=M(\mathbf{Q}, m) \vee M\left(\mathbf{Z}_{p^{\infty}}, m-1\right)$. It is easily seen that the space $E_{i}$ verifies conditions (1), (2), (3), (4) except the $i$ th, but $E_{i} \neq S_{p}^{m}$.

The above theorem shows that the cohomology with coefficients $\mathbf{Z}_{p}, \mathbf{Z}_{p}, \mathbf{Q}$ is not enough to characterize the localized spheres. The following theorem shows that the cohomology with coefficients $\mathbf{Z}_{(p)}$ is suitable for this purpose.

THEOREM 5. Let $E$ be a p-local nilpotent space such that $H^{*}\left(E ; \mathbf{Z}_{(p)}\right)=$ $H^{*}\left(S^{m} ; \mathbf{Z}_{(p)}\right)$. Then $E=S_{p}^{m}$.

Proof. From the universal coefficient theorem we obtain $\operatorname{Hom}\left(H_{r} E, \mathbf{Z}_{(p)}\right)=0$ if $r \neq m$ and $\operatorname{Ext}\left(H_{r} E, \mathbf{Z}_{(p)}\right)=0$ if $r \neq m-1$. Further, $\operatorname{Ext}\left(H_{m-1} E, \mathbf{Z}_{(p)}\right)$ is a $p$-local subgroup of $\mathbf{Z}_{(p)}$. Then either $\operatorname{Ext}\left(H_{m-1} E, \mathbf{Z}_{(p)}\right)=\mathbf{Z}_{(p)}$ or $\operatorname{Ext}\left(H_{m-1} E, \mathbf{Z}_{(p)}\right)=0$. But in the first case $\mathbf{Z}_{(p)}$ will be a cotorsion group (cf. [1, I, p. 232] and Theorem 54.6 of $\left[1, I\right.$, p. 235]) and this is impossible because $\operatorname{Ext}\left(\mathbf{Q}, \mathbf{Z}_{(p)}\right)=\mathbf{Q}^{\aleph_{0}} \neq 0$. Hence, $\operatorname{Ext}\left(H_{r} E, \mathbf{Z}_{(p)}\right)=0$ for all $r$.

Let $B_{r}$ be a $p$-basic subgroup of $H_{r} E$. If $B_{r}$ contains a direct summand of the form $\mathbf{Z}_{p^{k}}$ then it follows easily from the definition of $p$-basic subgroup that $\mathbf{Z}_{p^{k}}$ is a direct summand of $H_{r} E$. But $\operatorname{Ext}\left(\mathbf{Z}_{p^{k}}, \mathbf{Z}_{(p)}\right)=\mathbf{Z}_{(p)} / p^{k} \mathbf{Z}_{(p)} \neq 0$. Hence, $B_{r}$ is free. Let us consider the Hom-Ext exact sequence associated to $\mathbf{Z}_{(p)} \stackrel{\cdot p}{\rightarrow} \mathbf{Z}_{(p)} \rightarrow \mathbf{Z}_{p}$ : 


$$
0 \rightarrow \operatorname{Hom}\left(H_{r} E, \mathbf{Z}_{(p)}\right) \stackrel{\cdot p}{\rightarrow} \operatorname{Hom}\left(H_{r} E, \mathbf{Z}_{(p)}\right) \rightarrow \operatorname{Hom}\left(H_{r} E, \mathbf{Z}_{p}\right) \rightarrow 0 .
$$

We get that $\operatorname{Hom}\left(H_{r} E, \mathbf{Z}_{p}\right)=0$ if $r \neq m$. By Lemma $1, \operatorname{Hom}\left(B_{r}, \mathbf{Z}_{p}\right)=$ $\operatorname{Hom}\left(H_{r} E, \mathbf{Z}_{p}\right)=0$, hence $B_{r}=0$ if $r \neq m$. This shows that $H_{r} E$ is a divisible group for $r \neq m$. But the structure theorem for divisible groups and the equalities $\operatorname{Ext}\left(\mathbf{Q}, \mathbf{Z}_{(p)}\right)=\mathbf{Q}^{\aleph_{0}}, \operatorname{Ext}\left(\mathbf{Z}_{p^{\infty}}, \mathbf{Z}_{(p)}\right)=\hat{\mathbf{Z}}_{p}, \operatorname{Ext}\left(H_{r} E, \mathbf{Z}_{(p)}\right)=0$ yield $H_{r} E=0$ if $r \neq$ $m$.

In the case $r=m$ the above exact sequence shows that $\operatorname{Hom}\left(H_{m} E, \mathbf{Z}_{p}\right)=\mathbf{Z}_{p}$. By Lemma $1, B_{m}=\mathbf{Z}$. The Hom-Ext exact sequence associated to $\mathbf{Z} \mapsto$ $H_{m} E \rightarrow H_{m} E / \mathbf{Z}$ shows that $\operatorname{Ext}\left(H_{m} E / \mathbf{Z}, \mathbf{Z}_{(p)}\right)$ is the image of the countable group $\operatorname{Hom}\left(\mathbf{Z}, \mathbf{Z}_{(p)}\right)$. Since $\operatorname{Ext}\left(\mathbf{Q}, \mathbf{Z}_{(p)}\right)$ and $\operatorname{Ext}\left(\mathbf{Z}_{p^{\infty}}, \mathbf{Z}_{(p)}\right)$ are both uncountable and since $H_{m} E / \mathbf{Z}$ is a divisible group, we get that $H_{m} E / \mathbf{Z}$ is a torsion group without $p$-torsion. This leads to $\operatorname{Hom}\left(H_{m} E / \mathbf{Z}, \mathbf{Q}\right)=0$ and $\operatorname{so} \operatorname{Hom}\left(H_{m} E, \mathbf{Q}\right)=$ $\operatorname{Hom}(\mathbf{Z}, \mathbf{Q})=\mathbf{Q}$. Hence $H_{m} E$ is a group of torsion-free rank one. Further, $H_{m} E$ is torsion-free because $H_{m} E / Z$ has no $p$-torsion and $H_{m} E$ is $p$-local. Now we can show, as in the proof of Theorem 4 that $H_{m} E=\mathbf{Z}_{(p)}$ and so the space $E$ has the same integral homology as $S_{p}^{m}$. Then, by Lemma $3, E$ is a localized sphere $S_{p}^{m}$.

\section{REFERENCES}

1. L. Fuchs, Infinite abelian groups, Academic Press, New York, 1970.

2. P. Hilton, G. Mislin, and J. Roitberg, Localization of nilpotent groups and spaces, North-Holland Mathematics Studies 15, North-Holland, Amsterdam, 1975.

Secció de Matemàtiques, Universitat Autònoma de Barcelona, Bellaterra (Barcelona), SPAIN

Current address: Forschungs Institut für Mathematik, ETH-Zentrum, 8092-Zürich, Switzerland 